\title{
SUPERSYMMETRY SEARCHES AT THE COLLIDER DETECTOR AT FERMILAB
}

\author{
D. TSYBYCHEV \\ Physics Department, University of Florida, Gainesville, Fl 32611-8440, USA \\ E-mail: tsybych@phys.ufl.edu \\ FOR THE CDF COLLABORATION \\ This article presents the current experimental results of searches for Supersymme- \\ try (SUSY) at the Collider Detector at Fermilab (CDF), using over $110 p b^{-1}$ of \\ proton-antiproton collision data with $\sqrt{s}=1800 \mathrm{GeV}$ collected during the period \\ 1992-1995. Since no signal was found, limits on the production of supersymmetric \\ particles are derived. The prospects for supersymmetry searches at Run II of the \\ Tevatron, that began in March 2001, are also discussed here.
}

\section{Introduction}

\subsection{Supersymmetry phenomenology and experimental signatures}

Supersymmetry (SUSY) is a larger space-time symmetry that relates bosons and fermions, so that every Standard Model (SM) particle has a supersymmetric partner with spin difference $1 / 2$. In addition there are 2 Higgs doublets corresponding to 5 Higgs particles. Furthermore, new conserved quantum number is introduced to avoid Lepton(L) and/or Baryon (B) number violation. $R$ - parity is a multiplicative quantum number, defined as $R=(-1)^{3(B-L)+2 S}$ where $\mathrm{S}$ is the spin of the particle. For the SM particles $R=1$, whereas $R=-1$ for the SUSY partners. The important consequences are that SUSY particles would be produced in pairs in collider experiments, and decay to stable final state particles: the Lightest Supersymmetric Particle (LSP) and SM quarks and leptons. A comprehensive SUSY search is almost impossible since the Minimal Supersymmetric extension to the Standard Model (MSSM), with $\mathrm{R}$ parity conservation contains 124 truly independent parameters. So far none of the superpartners of the SM particles have been observed, implying that SUSY cannot be an exact symmetry of the nature. There are several models that are based on different mechanisms of spontaneous SUSY breaking. The masses, production cross-sections and decay branching ratios of supersymmetric particles are unknown and highly dependent on the particular SUSY breaking scenario, which eventually lead to different experimental signatures. Thus two different approaches can be adopted. The most general searches look for any deviations from the SM. In this approach all measurable signatures and phase space are scanned and the results can be interpreted based

proceeding: submitted to World Scientific on August 31, 2001 
on some SUSY model. However those searches are not maximally efficient for a particular model. An alternative approach is to search for specific signals suggested by particular models in which some theoretical assumptions are also made to reduce the number of free parameters.

The two models most often used to interpret experimental data are:

Minimal Super Gravity (mSUGRA). Supersymmetry breaking occurs at the GUT scale $\sim 10^{16} \mathrm{GeV}$. The LSP is the neutralino and experimental signatures depend on next lightest superparticle (NLSP) masses, and whether $R$-parity is conserved.

Gauge Mediated Supersymmetry breaking (GMSB). The SUSY breaking scale is around the electroweak scale. In this case the gravitino is light and becomes the LSP. The experimental signatures also depend on NLSP production, but final states can be photon enriched.

In most of SUSY models the LSP is assumed to be neutral and only weakly interacting, so its experimental signature is missing energy in the detector. In models where the $R$-parity is not conserved the LSP is not stable and can decay to SM particles. This introduces a variety of new signatures.

\subsection{The CDF detector}

In Run I, the CDF detector relied on a high resolution tracking system. It consisted of three tracking detectors enclosed in solenoidal superconducting magnet producing a $1.4 \mathrm{~T}$ field. The innermost component was a silicon microstrip detector, which could detect displaced vertices from B-meson decays. Outside the microstrips was a time projection chamber which allowed tracking in $r-z$ plane. This was enclosed in an open-cell drift chamber which measured charged particle momentum for particles with $|\eta|<1$. Hadronic and electromagnetic sampling calorimeters were located outside the magnet, arranged in a projective tower geometry. A system of muon detectors was installed outside the calorimeter with coverage $|\eta|<1$.

\section{Signature-based searches}

\subsection{Jets and Missing Energy Search for Squarks and Gluinos.}

Since gluino and squarks interact strongly, their production cross section could be large at the Tevatron, compared with other sparticles. Decays of squarks and gluinos depend on their electroweak couplings to charginos/neutralinos and their masses. If the squark is heavier than the gluino, models typically predict $\tilde{q} \rightarrow q \tilde{g}$ and $\tilde{g} \rightarrow q q \tilde{\chi}_{1}^{0}$. If the gluino is heavier then $\tilde{g} \rightarrow q \tilde{q}$ and

proceeding: submitted to World Scientific on August 31, 2001 
$\tilde{q} \rightarrow q \tilde{\chi}_{1}^{0}$. The $\chi_{1}^{0}$ escapes the detector and produces large missing transverse energy $\left(\not_{t}\right)$. In this case the signature is jets and $\not_{t}$.

This analysis ${ }^{1}$ used the so-called "blind box" approach. The signal region was defined a priori using several kinematic variables in the phase space where the SUSY signature is most likely to be: $E_{t}$, the sum of the transverse energies of 2 jets and $E_{t}\left(H_{t}=E_{t}^{2^{n d} j e t}+E_{t}^{3^{\text {rd }} j e t}+E_{t}\right)$, and the number of isolated tracks in the event $\left(N_{t r k}^{i s o}\right)$. The "blind box" was defined by requiring $\mathbb{E}_{t}>70 \mathrm{GeV}, H_{t}>150 \mathrm{GeV}, N_{t r k}^{i s o}=0$. Events with large $\mathbb{E}_{t}$ and $\geq 3$ jets are expected primarily from QCD, $Z(\rightarrow \nu \nu)+\geq 3$ jets, $W(\rightarrow \tau \nu)+\geq 2$ jets ( the third jets is due to hadronic $\tau$ decay) and $t \bar{t}$ production. The SM backgrounds in the data were studied and normalized to MC outside the signal region. The "blind box" data were inspected only after the entire search path had been defined and selections optimized to increase sensitivity to the SUSY signatures.

The SM predictions in the "blind box" were $76 \pm 13$ events and 74 events were observed in $84 \mathrm{pb}^{-1}$ data. The derived $95 \%$ C.L. excluded region is shown on the $m_{\tilde{q}}-m_{\tilde{g}}$ plane in Figure 1 . The search excludes gluino mass below $195 \mathrm{GeV} / c^{2}$, independent of the squark mass. For the case $m_{\tilde{q}} \approx m_{\tilde{g}}$, gluino masses below $300 \mathrm{GeV} / c^{2}$ are excluded.
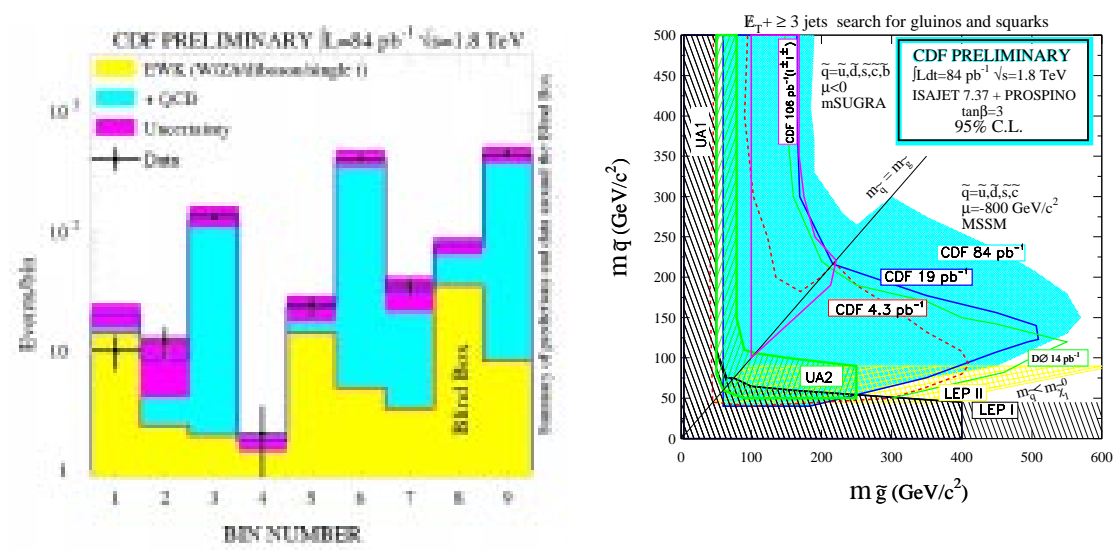

Figure 1. CDF result on squark-gluino blind search in jets $+E_{t}$. On the left is the comparison of SM prediction and data in bins around the blind box. On the right is the $95 \%$ C.L. limit curve in the $m_{\tilde{q}}-m_{\tilde{g}}$ plane for $\tan \beta=3$. Results from the previous searches are also shown. 


\subsection{Search for new physics in photon-lepton events}

This analysis was inspired by the unexpected eer $\gamma \quad E_{t}$ event observed at $\mathrm{CDF}^{2}$ and thus searched for anomalous production of events with a high $E_{t}$ photon and a lepton $l(e$ or $\mu)$ in the final state.

All the $\gamma-l$ events are categorized by the number of final state particles:

Two body: 1 photon, 1 lepton and no additional photons and leptons. The azimuthal angle between lepton and photon was required to be more than $150^{\circ}$.

Multi body: This category included 1 photon, 1 lepton and $\not_{t}>25 \mathrm{GeV}$ or additional lepton (photon), and was further divided into three different subsets.

SM backgrounds for lepton-photon events come from $W / Z+\gamma$ production, $l+$ jet production, where jet was misidentified as a photon, and $b / c$ semileptonic decays. The event count in each of the subcategories were compared with SM background. The preliminary results of the analysis are shown in Table 1. This analysis sets up an important Run II a priori search.

Table 1. Preliminary CDF photon-lepton results for analyzed categories, including the number of events $N_{S M}$ predicted by the SM, the number $N_{0}$ observed in CDF data, and the probability that $N_{S M}$ can fluctuate to $N_{0}$

\begin{tabular}{|c|c|c|c|}
\hline Category & Predicted $N_{S M}$ & Observed $N_{0}$ & $\mathrm{P}\left(N \geq N_{0} \mid N_{S M}\right), \%$ \\
\hline All $l \gamma X$ & - & 77 & - \\
\hline Two-Body $l \gamma$ & $24.9 \pm 2.4$ & 33 & 9.3 \\
\hline Multi-Body $l \gamma X$ & $20.2 \pm 1.7$ & 27 & 10.0 \\
\hline Multi-Body $l l \gamma X$ & $5.8 \pm 0.6$ & 5 & 61.0 \\
\hline Multi-Body $l \gamma \gamma X$ & $0.02 \pm 0.02$ & 1 & 1.5 \\
\hline Multi-Body $l \gamma E_{t} X$ & $7.6 \pm 0.7$ & 16 & 0.7 \\
\hline
\end{tabular}

\section{The MSSM searches in Run I}

\subsection{Searches for a light stop}

Due to the large top quark mass the left and right components of the SUSY partner to the top can mix, resulting in one light and one heavy mass eigenstate. If this occurs, then the light stop could by the second lightest SUSY particle and much lighter than other squarks. Depending on the masses of other sparticles such as charginos, neutralinos and sneutrinos, different signatures could arise from the decay of stops, including $\tilde{t} \rightarrow c \tilde{\chi}_{1}^{0}, \tilde{t} \rightarrow b \tilde{\chi}_{1}^{ \pm}$and $\tilde{t} \rightarrow b l \tilde{\nu}$

The search for stop in $\tilde{t} \rightarrow c \tilde{\chi}_{1}^{0}$ decay mode ${ }^{3}$ looked for 2 or 3 acolinear jets with at least $40 \mathrm{GeV}$ of missing $E_{t}$. Events with identified leptons were 
removed, and then jets originating from decay of charm quarks were identified using the displaced tracks reconstructed by silicon detector. Eleven events were observed with $14.5 \pm 4.2$ events expected from SM background coming primarily from $W / Z+$ jets and QCD heavy quark production. The $95 \%$ C.L. exclusion contour is shown on Figure 2.

Another search looked for the stop in the $b l \not_{t}$ signature ${ }^{4}$. Two separate $\tilde{t}$ decay modes were considered $\tilde{t} \rightarrow b \tilde{\chi}_{1}^{ \pm}$, with chargino subsequent decay into $l^{ \pm} \nu \tilde{\chi}_{1}^{0}$ and $\tilde{t} \rightarrow b l \tilde{\nu}$, dominant whenever the sneutrino is light and $\tilde{t} \rightarrow b \tilde{\chi}_{1}^{ \pm}$ is not kinematically allowed. This analysis relied on the excellent b-tagging ability of the CDF silicon detector. Events were selected by requiring of at least one $e$ or $\mu$ with $p_{t}>10 \mathrm{GeV} / \mathrm{c}$, two or more jets with energies $E_{t}^{1^{s t}}{ }^{\text {jet }}>12 \mathrm{GeV}, E_{t}^{2^{\text {nd }} \text { jet }}>8 \mathrm{GeV}$ ( jets were ordered by energy) and large $\mathbb{E}_{t}>25 \mathrm{GeV}$ with $\Delta \phi\left(\mathbb{E}_{t}\right.$, jet $)>0.5$. At least one jet had to be identified as a b-jet candidate. There were 81 events observed in data, with $86.2 \pm 5.2$ expected from the SM background and fake leptons. The resulting excluded region $\left(m_{\tilde{t}}\right.$ versus $\left.m_{\tilde{\nu}}\right)$ for $\tilde{t} \rightarrow b l \tilde{\nu}$ is given in Figure 2.
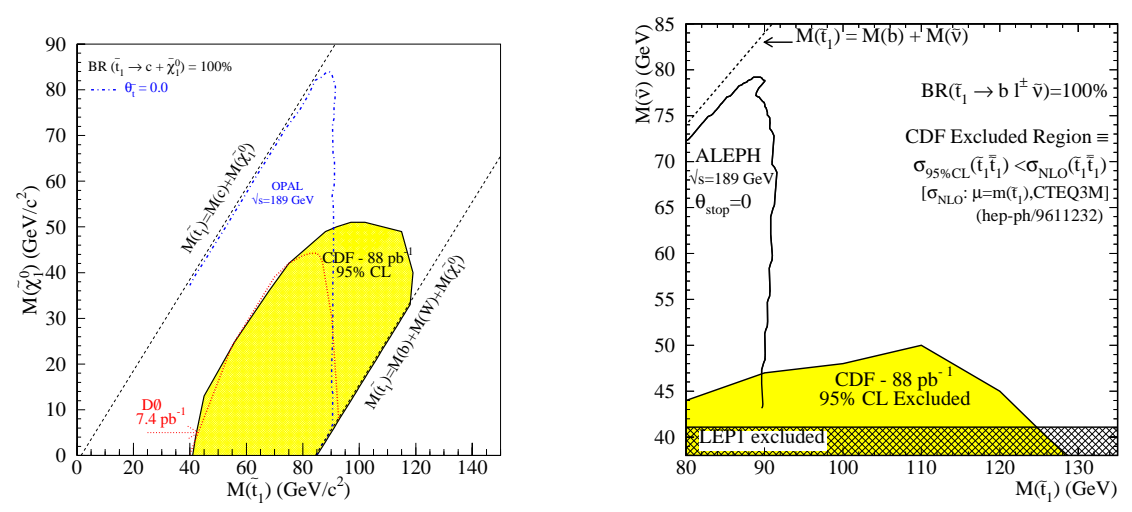

Figure 2. CDF results on stop quark search. On the left is excluded region at 95\% C.L. for stop from $\tilde{t} \rightarrow b \tilde{\chi}_{1}^{0}$. On the right is the excluded region from $\tilde{t} \rightarrow b l \tilde{\nu}$ analysis.

\subsection{Search for squarks and gluinos using LS dileptons}

In addition to the classic $E_{t}+$ jets search, CDF looked for gluinos decaying through charginos or neutralinos, which decay to leptons with primarily uncorelated charge, giving the like sign (LS) lepton signature ${ }^{5}$. Events were selected by asking for an isolated central lepton with $E_{t}^{l_{1}}>11 \mathrm{GeV}$ and a second lepton with $E_{t}^{l_{2}}>5 \mathrm{GeV}$. To reject background, leptons were required 
to be well separated in $\eta-\phi$ space. Events were also required to have two jets with $E_{t}>15 \mathrm{GeV}$ and $E_{t}>25 \mathrm{GeV}$. A like-sign requirement on the dilepton events was applied. No candidate event was observed, which is consistent with SM background expectations from $t \bar{t}, b \bar{b}$, diboson and Drell-Yan processes. The results of the analysis are shown in Figure 3. The limits are interpreted for a model that assumes $\tan \beta=2, \mu=-800 \mathrm{GeV} / c^{2}, m_{A_{0}}=500$ $\mathrm{GeV} / c^{2}$. The search excludes gluino masses below $168 \mathrm{GeV} / c^{2}$, independent of the squark mass. For the case $m_{\tilde{q}} \approx m_{\tilde{g}}$, gluino mass below $221 \mathrm{GeV} / c^{2}$ are excluded.

\subsection{Gaugino searches in trilepton signatures}

The trilepton mode is one of the cleanest signatures for supersymmetry at the Tevatron ${ }^{6}$. It could originate from the associated production of the lightest chargino $\tilde{\chi}_{1}^{ \pm}$and the second-lightest neutralino $\tilde{\chi}_{2}^{0}$ followed by decays $\tilde{\chi}_{1}^{ \pm} \rightarrow l^{ \pm} \nu \tilde{\chi}_{1}^{0}$ and $\tilde{\chi}_{2}^{0} \rightarrow \tilde{\chi}_{1}^{0} l^{+} l^{-}$, giving the signature of three leptons and $\not_{t}$.

For this search, the first lepton had to pass tight identification cuts, and have $E_{t}>11 \mathrm{GeV}$. The other two leptons were required to pass looser cuts and have $E_{t}>5 \mathrm{GeV}$. Mass cuts around $Z, J / \Psi$ and $\Upsilon$ were imposed to remove SM background. After requiring $E_{t}>15 \mathrm{GeV}$, no events were observed. For the case $\tan \beta=2, \mu=-600 \mathrm{GeV} / c^{2}, m_{\tilde{q}}=m_{\tilde{g}}$, chargino masses below $81.5 \mathrm{GeV} / c^{2}$ and second neutralino masses below $82.2 \mathrm{GeV} / c^{2}$ were excluded. The CDF limits on the chargino mass from the trilepton search are shown in Figure 4.

\subsection{Light gravitino searches}

GMSB models predict that the gravitino $(\tilde{G})$ is the LSP, and the other sparticles are much heavier and hence are inaccessible at the Tevatron. The production cross-section of gravitinos pairs is inversely proportional to $M_{\tilde{G}}$, giving significant cross sections for gravitino masses below $10^{-5} \mathrm{eV} / c^{2}$. On the other hand, the gravitino mass is proportional to F, the SUSY-breaking scale. At the Tevatron the following processes could occur $q \bar{q} \rightarrow \tilde{G} \tilde{G} g, q g \rightarrow \tilde{G} \tilde{G} q$, $q \bar{g} \rightarrow \tilde{G} \tilde{G} \bar{q}, g g \rightarrow \tilde{G} \tilde{G} g$. The signature for this search is then large $\mathbb{E}_{t}$ and a high $E_{t}$ jet ${ }^{7}$. The $Z_{t}$ spectrum from the data along with SM background for this analysis is shown in Figure 5, after all requirements except for the cut on $E_{t}$. After applying a $\mathbb{E}_{t}>200 \mathrm{GeV}$ cut, no excess was observed and an upper limit was set on the production cross-section for $p_{t}(\tilde{G} \tilde{G})>100 \mathrm{GeV} / \mathrm{c}$ at $93 \mathrm{fb}$, which was translated to gravitino mass $m_{\tilde{G}}>1.2 \times 10^{-5} \mathrm{eV} / c^{2}$ and a SUSY-breaking scale limit $\sqrt{F}>221 \mathrm{GeV}$.

proceeding: submitted to World Scientific on August 31, 2001 

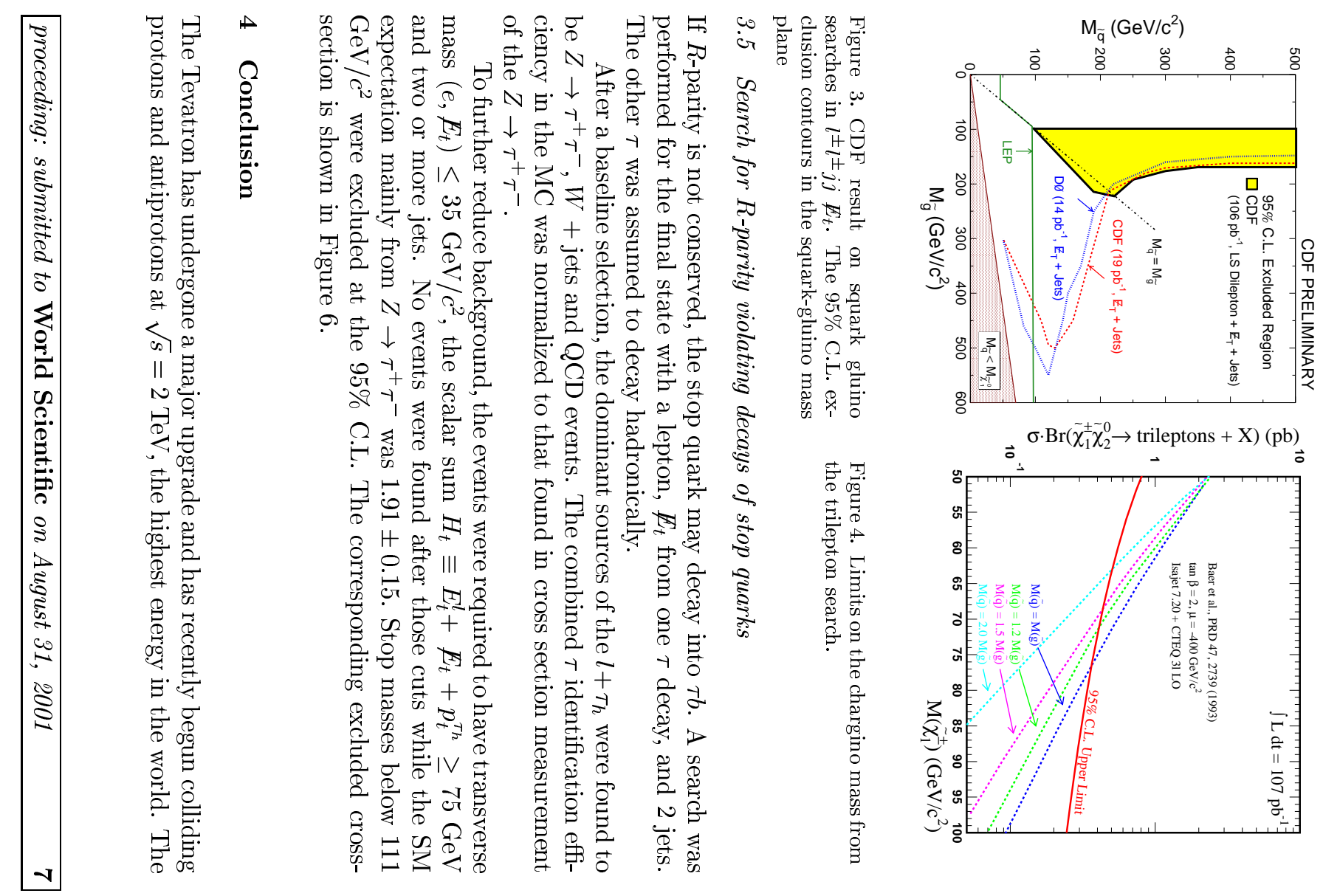


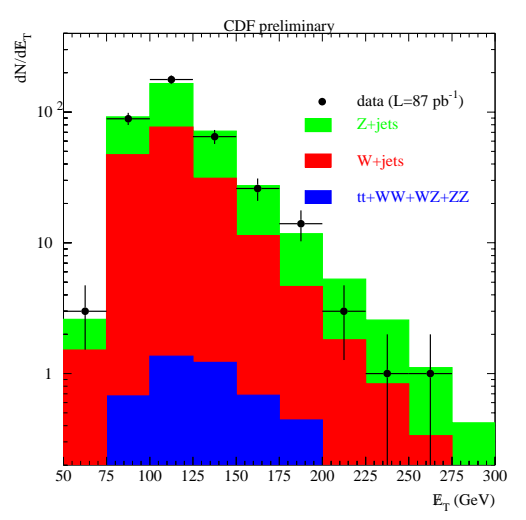

Figure 5. The $E_{t}$ spectrum in the search for a light gravitino.

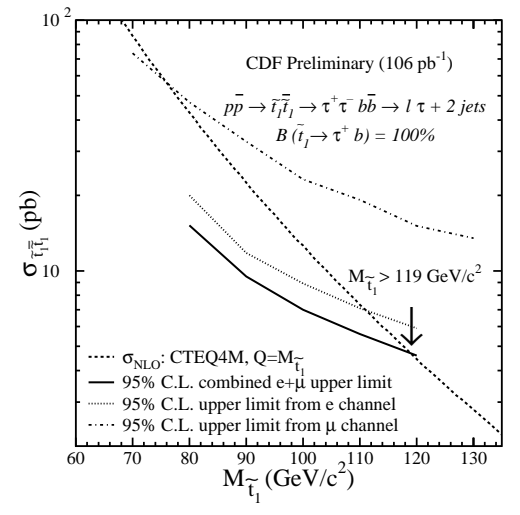

Figure 6. The upper limit on the $\tilde{\tilde{t}} \overline{\tilde{t}}$ production cross-section at $95 \%$ C.L. from $R$ parity violation stop decay to $\tau b$

CDF detector was also upgraded for the Run II. Among other systems it has now a new silicon tracking detector with extended coverage,a new end-plug calorimeter,a time of flight detector to improve particle identification and extended coverage in the muon system.

In conclusion, the CDF experiment has conducted extensive searches for SUSY in Run I at the Fermilab Tevatron. No evidence has been found so far. The initial Run II dataset will consist of at least $2 \mathrm{fb}^{-1}$, twenty times more data than collected in Run I. The CDF and D0 experiments have a significant discovery potential for the Supersymmetry before the LHC begins to operate in 2007 .

\section{References}

1. T. Affolder et al, Submitted to Phys. Rev. Lett..

2. F. Abe et al, Phys. Rev. D 59, 092002 (1999)

3. T. Affolder et al, Phys. Rev. Lett. 84, 5704 (2000).

4. T. Affolder et al, Phys. Rev. Lett. 84, 5273 (2000).

5. T. Affolder et al, hep-ex/0106061 Submitted to Phys. Rev. Lett..

6. F. Abe et al, Phys. Rev. Lett. 80, 5275 (1998).

7. T. Affolder et al, Phys. Rev. Lett. 85, 1378 (2000). 